\title{
Common Mistakes in Creating Sentences Consisting of a Subject and Predicate and in Translating them to Tamil: A Case Study of Undergraduates of the Department of Arabic Language of South Eastern University of Sri Lanka الأخطاء الشائعة في إنشاء الجمل المتكونة من مبتدإ وخبر وترجمتها إلى اللغة التاملية: دراسة حالة لطلبة

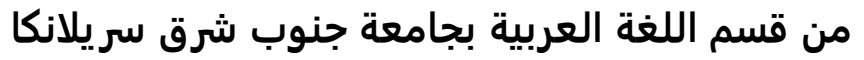

M.H.A. Munas ${ }^{1} \bowtie$ and M.S. Zunoomy ${ }^{2}$

South Eastern University of Sri Lanka, Oluvil, Sri Lanka

Corresponding Author: Hakeem Salmon, E-mail: munas@seu.ac.lk

\section{ARTICLE INFORMATION}

Received: 02 October 2021

Accepted: 25 November 2021

Published: 24 December 2021

DOI: 10.32996/ijllt.2021.4.12.17

\section{KEYWORDS}

Errors, Creating sentence, nominal sentence, Translation, Arabic, Tamil

\section{ABSTRACT}

Nominal sentence is a specific aspect of a language. It consists of a subject and predicate. The predicate reflects the subject. Second language learners of Arabic at the level university face difficulties in creating sentences consisting of a subject and predicate and in translating them into Tamil. At the same time, they have learned Arabic grammar in Sri Lankan Arabic Colleges with the $5-7$ years and also Tamil is their native language. Thus, this research aims to identify the grammar errors when writing nominal sentences and to translate them to Tamil and to rely on the reasons for them. To this, the research uses descriptive analytical methodology through a quantitative approach. It uses a questionnaire for primary data among the undergraduates of the Department of Arabic Language, South Eastern University of Sri Lanka. At the same time, secondary data were gathered from researches, books, articles, website articles. The research finds that the undergraduate has the enough theoretical knowledge about the nominal sentence and they have no confuse to develop a nominal sentence. At the same time, in the practical part, they are neutral level in writing a nominal sentence, in finding the error from them a, and in translating them into Tamil. Hence, the practical part is difficult for the undergraduates than the theoretical part.

الملخص:

الجملة الاسمية هي التي تبدأ بها الجمل في التقارير وفي الأخبار وفي الأحداث، وهي من أذبأ أهم

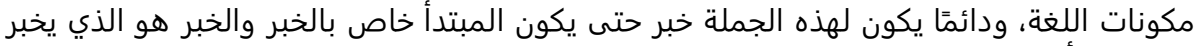

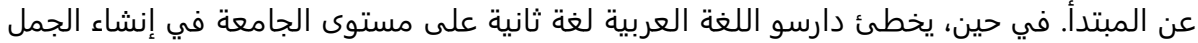

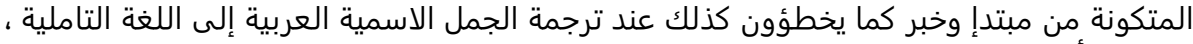

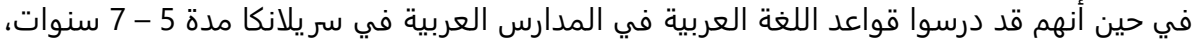

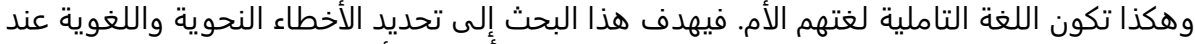

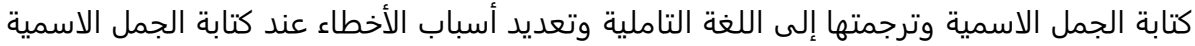

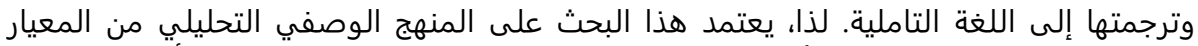

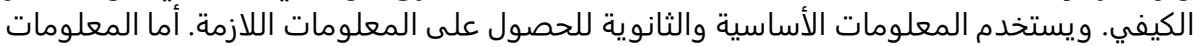

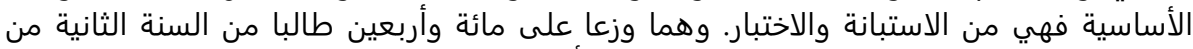

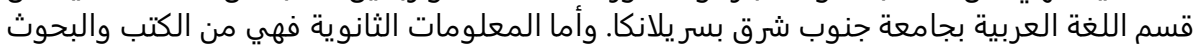

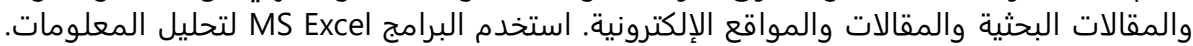

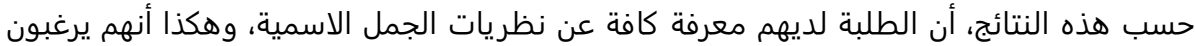

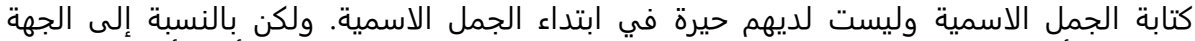

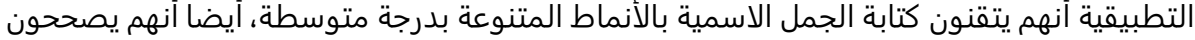

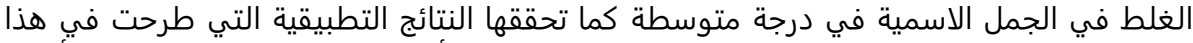

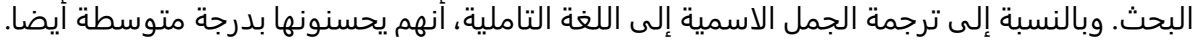

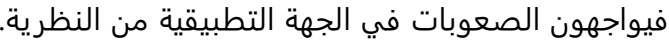

الكلمات المفتاحية: الأخطاء، إنشاء الجملة، الجملة الاسمية، الترجمة، اللغة العربية 


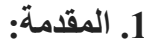

إن كلمة اللغة لها معنيان: معنى لغوي؛ و هو ما تسجله معاجم اللغة، وآخر اصطلاحي؛ و هو ما ينقن عليه بين العلماء و الجمهور أو بين العلماء فقط.

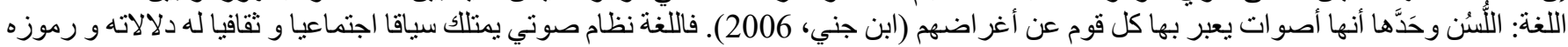
و هو قابل للنمو التطور يخضع ذللك للظروف التاريخية والحضارية التي يمر بها المجنمع (علي الصفحي، 2010).

يبدو أن ارتباط الإسلام باللغة العربية ذلك الارتباط الوثيق الذي يمثل في القر آن الكريم والأحاديث النبوية قد جعل للغة العربية مكانة مرموفة على غير ها فيا

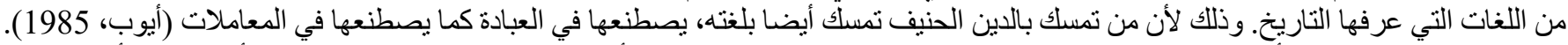

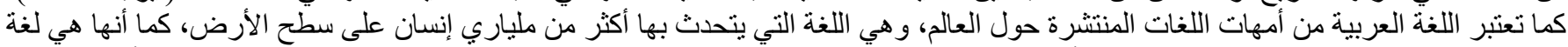

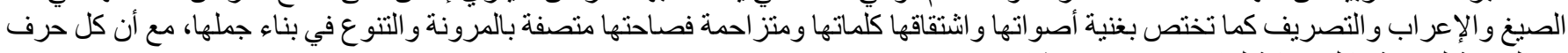
في العربية له قيمة وكل حركة لها هدف خاص (هشام، 2017).

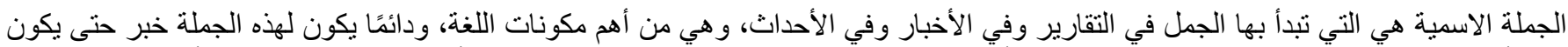

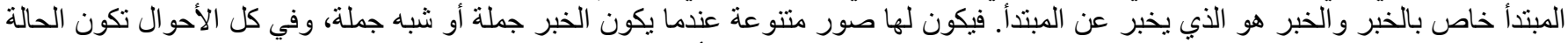

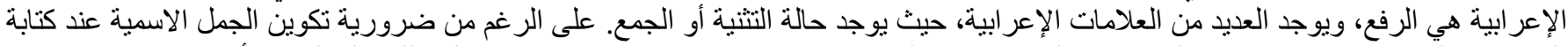

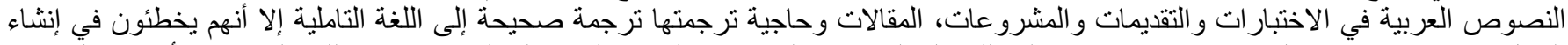

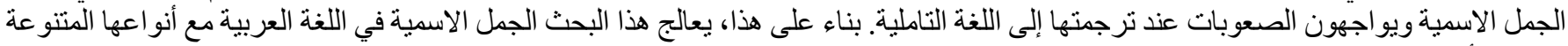
لتحديد الأخطاء التي تحدث عند كتابتها وهكذا الصعوبات التي تقع عند ترجمتها إلى اللى اللغة التاملية.

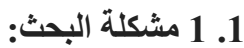

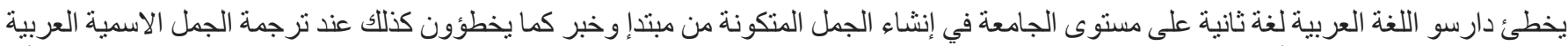

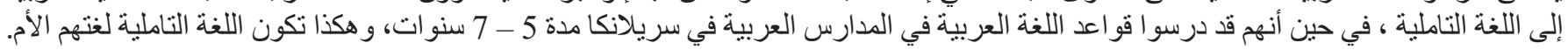

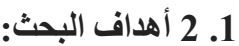

1. ت تحديد الأخطاء النحوية واللغوية عند كتابة الجمل الاسمية وترجمتها إلى اللغة التاملية.

2. ت تعديد أسباب الأخطاء عند كتابة الجمل الاسمية وترجمتها إلى اللغة التخاملية.

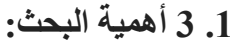

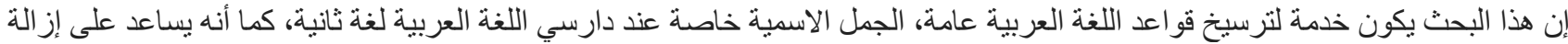

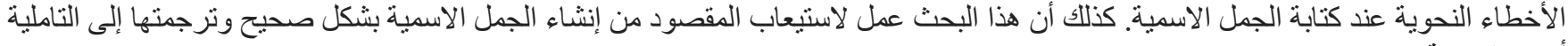

أحسن ترجمة.

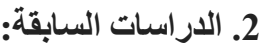

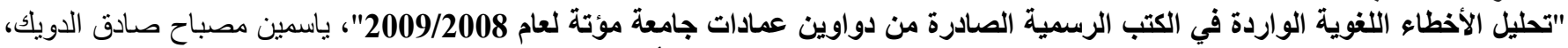

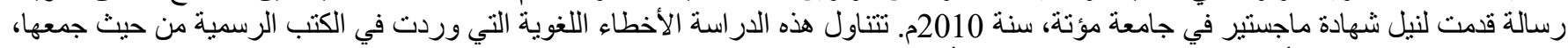

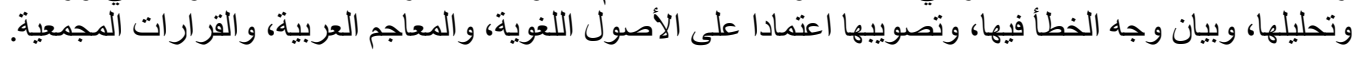

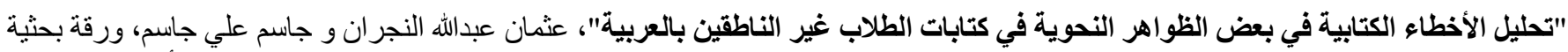

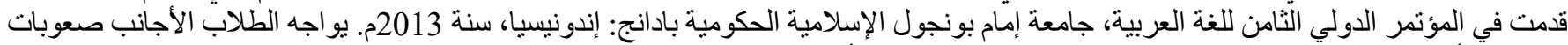

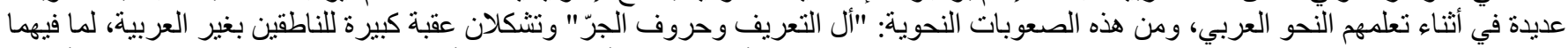

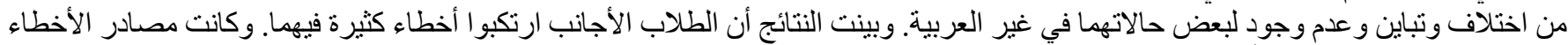
كثيرة ومنها؛ النقل اللغوي و الأسباب النطورية.

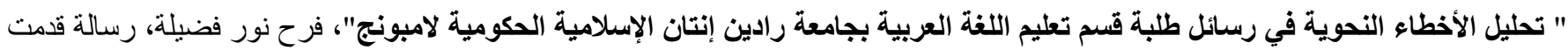

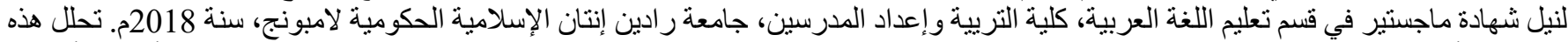

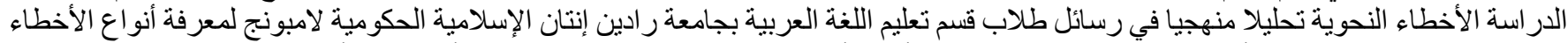

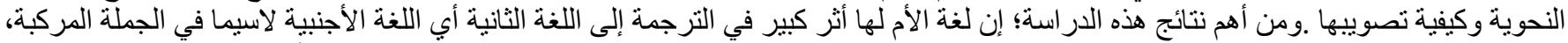

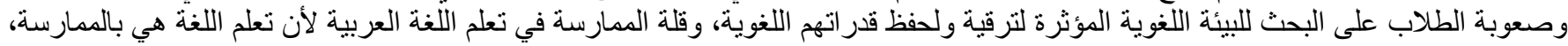

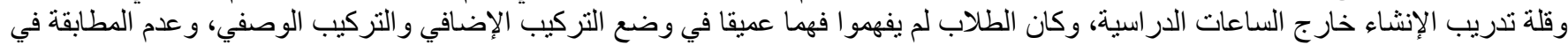

وضع الجملة الاسمية في غير مكانتها لمطابقتها في الجملة الفعلية.

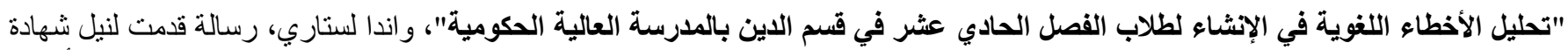

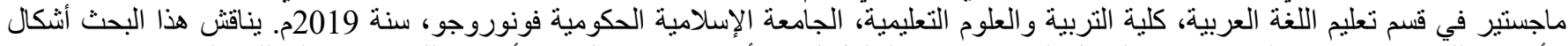

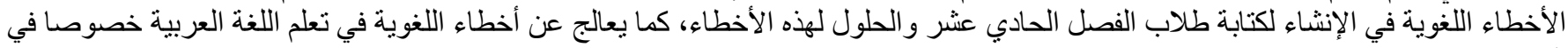




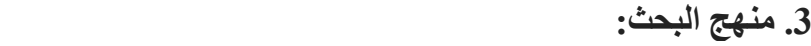

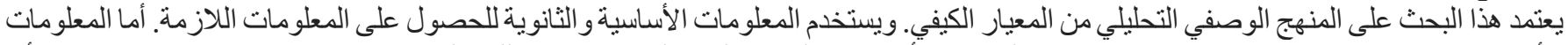

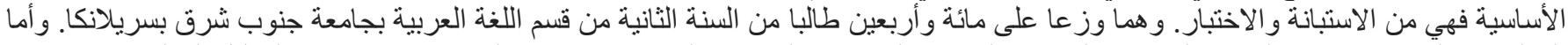

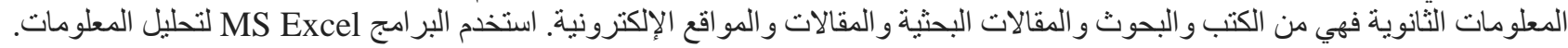

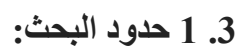
الحد البشري؛ طلبة السنة الثانية من دارسي اللغة العربية لغة ثانية بقسم اللغة العربية من جامعة جنوب شرق سريلانكا. الحد الموضوعي: إنشاء الجمل المتكونة من مبتدإوخبر (الجملة الاسمية) وترجمتها إلى اللغة التاملية. 4. 1 أنتائج والمناقثة:

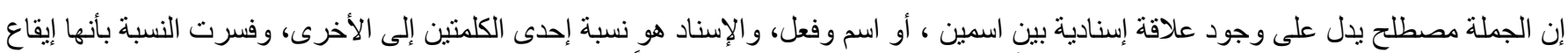

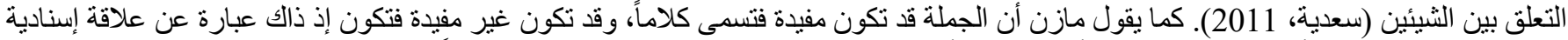

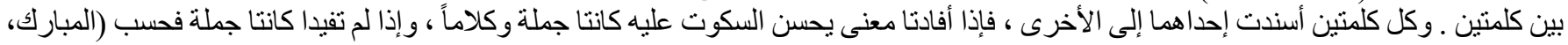

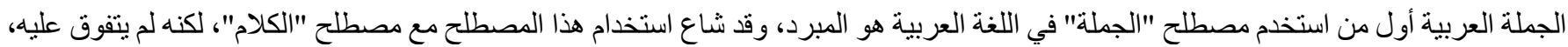

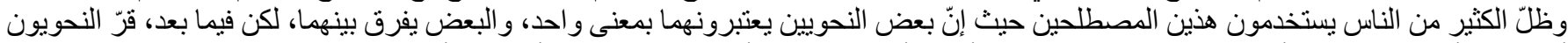
أن الجملة أعم من الكلام، لأن الإسناد في الجملة قد يكون أصليًا أو قد لا يكون، أما في الكلام فيجب أن يكون أصليًا (عبد اللطيف، 2003).

الجملة الاسمية

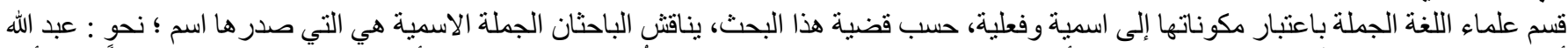

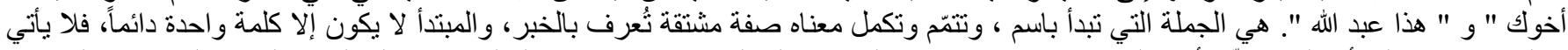

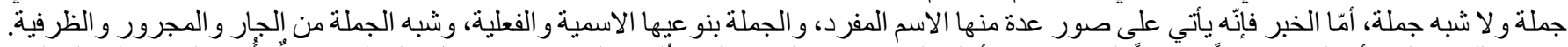

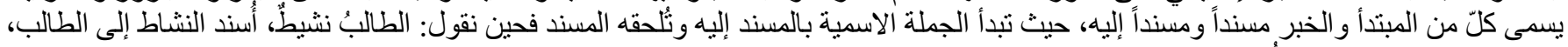

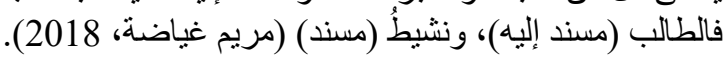

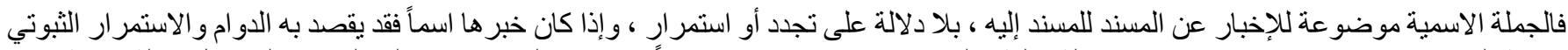

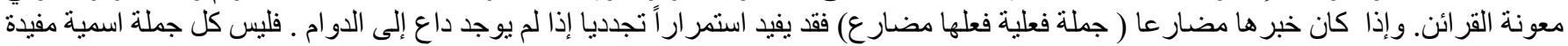

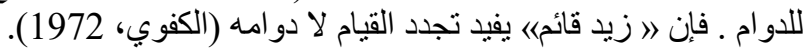

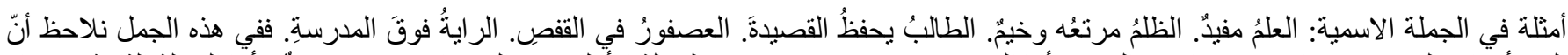

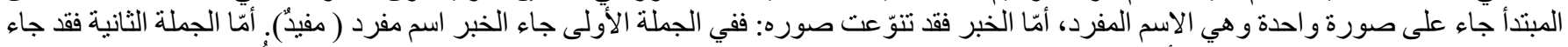

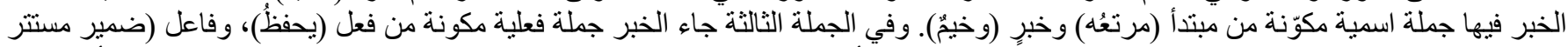

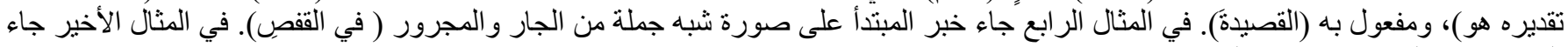
الخبر شبه جملة ظرفية (فوله وفَ المدرسة).

الأخطاء اللغوية

أما الأخطاء فهي تحدث عند ما يخر ج متعلم اللغة على قاعدة من القو اعد التي تحكم النظام اللغوي المعين مثل عدم التزام امه بنظام الجملة في اللغة العربية.

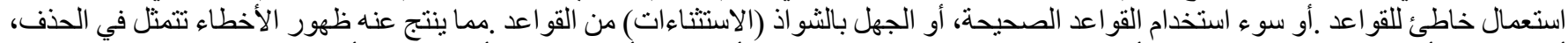

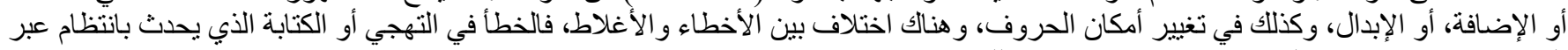

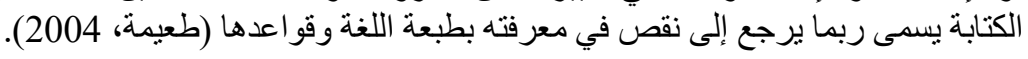

الأخطاء يقصد بها الأخطاء اللغوية أي انحر اف عما هو مقبول في اللغة العربية حسب المقاييس التي يتبعها الناطقون بالعربية الفصحى (طعيمة، 2004). أنواع الأخطاء اللغوية؛؛ الأخطاء النحوية: يقصد بالأخطاء النحوية :الأخطاء التي تتناول موضو عات النحو :كالتذكير، و التأنيث، و الإفر اد، و التثنية، و الجمع، و غير ها.

الأخطاء الصرفية: يقصد بالأخطاء الصرفية :هي الأخطاء التي تتناول موضو عات الصرف، كالتصغير، و النسب وغير ها الأخطاء الصوتية: يقصد بالأخطاء الصوتيّة :هي الأخطاء التي تقع في أصوات اللغة العربية وحر كاتها وما يعتريها من حذف، و إضافة، وإبدال، وغير ها. 
الأخطاء الإملائية: يقصد بالأخطاء الإملائية :الأخطاء التي تكون في كتابة الكلمة بشكلة غير صحيح أو مضبوط.كزيادة حرف، أو حذفه، أو إبداله، أو وضعه في غير موضعاء من الكلمة (فضيلة، 2018). بناء على هذا، أن هذا البحث يعالج الأخطاء التي تقع عند كتابة الجملة الاسمية وترجمتها لدى الطلبة در اسي اللغة العربية لغة ثانية. نتائج البحث: إن الجزء الأول من الاستبانة يسعى إلى تحديد مستو اهم النظري عن الجملة الاسمية ومكوناتها خلال هذه الأسئلة التي تتضمن عن ماهية الجملة الاسمية؛ ما تفهم عن الجملة الاسمية؛ الأبنة هل تو افق على وجود الجملة الاسمية في اللغات الأخرى غير اللغة العربية؟ إن للجملة الاسمية ركنين ، ما هما؟

\begin{tabular}{|c|c|c|c|c|}
\hline النسبة المئوية & الجواب الخاطئ & النسبة المئوية & الجواب الصحيح & النظريات \\
\hline$\% 6.2$ & 2 & $\% 3.97$ & 73 & ما تفهم عن الجملة الاسمية؟ \\
\hline$\% 4.17$ & 13 & $\% 6.82$ & 62 & إن للجملة الاسمية ركنين ، ما هما؟ \\
\hline$\% 16$ & 12 & $\% 84$ & 63 & اللغات الأخرى على وجير اللغة الجملة الاسمية في في \\
\hline
\end{tabular}

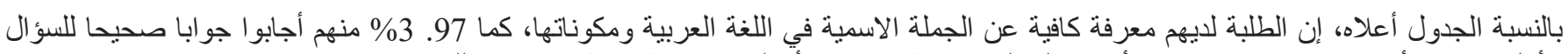
الأول، و هكذا، أن 82. 62\% منهم يعرفون أركان الجملة الاسمية، في حين أن لديهم معرفة كافية عن سائر اللغية اللغات. قد طرحت هذه الأسئلة لمعرفة رغباتهم وحالاتهم في إنشاء الجملة الاسمية. يتضحها الجدول التالي:

\begin{tabular}{|c|c|c|c|c|}
\hline النسبة المئوية & $\bar{\gamma}$ & النسبة المئوية & نعم & الأسئلة \\
\hline$\% 76$ & 57 & $\% 24$ & 18 & هل تشعر بالصعوبة في إنشاء الجملة الاسمية؟ \\
\hline$\% 16$ & 12 & $\% 84$ & 63 & في إنشاء الجملة الاسمية؛ الأنطاء التي تصدر كثير ا \\
\hline$\% 67$ & 50 & $\% 33$ & 25 & هل للك حيرة في ابتداء الجملة الاسمية ؟ \\
\hline
\end{tabular}

إن 24\% من الطلبة بشعرون بالصعوبة في إنشاء الجملة الاسمية، كما ليست لدي 33\% منهم حيرة في ابتداء الجمل الاسمية، وهكذا ممكن لهم أن بميزوا الأخطاء التي تصدر كنير البة في إنشاء الجملة الاسمية.

بعد معرفة الطلبة النظرية عن الجملة الاسمية ور غباتهم، يسعى هذا البحث إلى تحديد مستو اهم التطبيقية في تكوين الجملة الاسمية خلال الأسئلة الآتية؛

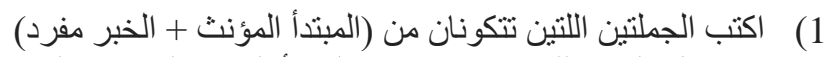

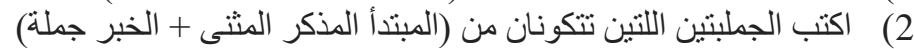

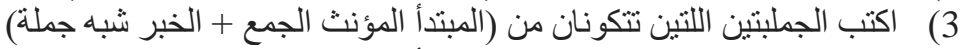

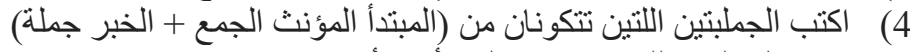
5) اكتب الجملتين اللتين تتكونان المبندأ من أسماء الإشارة.

قد كتب الطلبة كلهم 750 جملة حسب الأسئلة المطلوبة منهم. عند تصحيح أجوبة الطلبة للأسئلة المذكورة أعلاها مستخرجا الأخطاء النحوية الثنائعة التي وقع فيها كل طالب، قد تم حصول الجدول الآتي حسب نوع الجمل الصحيحة و الخاطئة ونسبتهما المئوية.

\begin{tabular}{|c|c|c|c|c|}
\hline النسبة المئوية & الجملة الخاطئة & النسبة المئوية & الجملة الصحيحة & أنماط الجملة \\
\hline$\% 21$ & 32 & $\% 79$ & 118 & المبندأ المؤنث + الخبر مفرد \\
\hline$\% 59$ & 88 & $\% 41$ & 62 & المبتدأ المذكر المثنى + الخبر جملة \\
\hline$\% 37$ & 56 & $\% 63$ & 94 & المبتدأ المؤنث الجمع + الخبر شبه جملة \\
\hline$\% 63$ & 94 & $\% 37$ & 56 & المبتدأ المؤنث الجمع + الخبر جملة \\
\hline$\% 39$ & 58 & $\% 61$ & 92 & المبتدأ من أسماء الإشارة \\
\hline
\end{tabular}

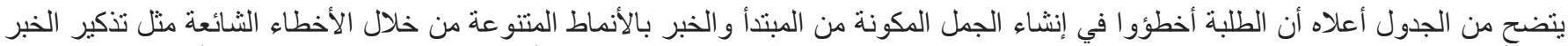

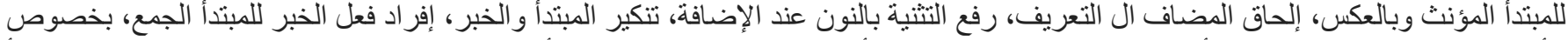
الأخطاء الإملائية. إن 79\% منهم أتقنو ا في إنشاء الجمل من (المبتدأ المؤنث + الخبر مفرد)، كما أتقن 63\% و 61\% منهم في إنشاء الجمل من (المبتدأ 


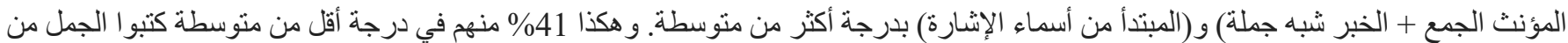
(المبندأ المذكر المثنى + الخبر جملة)، وأقل منهم 37\% كتبوا الجمل من (المبندأ المؤنث الجمع + الخبر جملة)، ومن الجدير بذكر أنهم أخطؤو ا كثير ا في من

تاليا، يحاول هذا البحث إلى كثف الغلط في الجمل الاسمية. لحصول هذا الهدف، طرحت ثمانية جمل التي تتكون الأخطاء النحوية كالتالي: 1 2

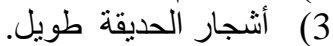

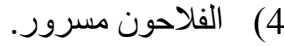
(5

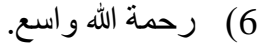
7) (6 أزهار الجنينة متفتحات. 8) بنات المدير ذاهبة إلى الجامعة. عند تصحيح أجوبة الطلبة للأسئلة المذكورة أعلاها مستخرجا الجمل المصححة وغير المصححة، قد نم حصول الجدول الآتي حسب المصحح وغير المصحح ونسبتهما المئوية.

\begin{tabular}{|c|c|c|c|c|}
\hline النسبة المئوية & غير المصحَّح & النسبة المئوية & المصحَّح & الجملة الخاطئة \\
\hline$\% 55$ & 41 & $\% 45$ & 34 & في هذا بيت أنثاث. \\
\hline$\% 51$ & 38 & $\% 49$ & 37 & ابنا سالم طبيب. \\
\hline$\% 23$ & 17 & $\% 77$ & 58 & أشجار الحديقة طويل. \\
\hline$\% 25$ & 19 & $\% 75$ & 56 & الفلاحون مسرور. \\
\hline$\% 16$ & 12 & $\% 84$ & 63 & 5 (5ما صديق. \\
\hline$\% 9$ & 7 & $\% 91$ & 68 & رحمة الله واسع. \\
\hline$\% 52$ & 39 & $\% 48$ & 36 & أزهار الجنينة متفتحات. \\
\hline$\% 47$ & 35 & $\% 53$ & 40 & بنات المدير ذا هبة إلى الجامعة. \\
\hline
\end{tabular}

بالنسبة الجدول المذكور أعلاه، قد صحح الطلبة الغلط في الجمل بدرجة متوسطة، لأنهم لم بعرفون ماهية الأخطاء الموجودة في الجمل المطروحة. ولذا

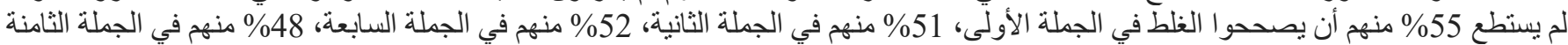
إلا بدرجة متوسطة. وفي حين أغلبيتهم صححو ا بدجة عالية تصحيحا تاما في الجملة الثالثة و الر ابعة و الخأمسة و السادسة. وفي ما يأتي، يسعى هذا البحث أن يحدد الترجمة الصحيحة و الخاطئة للجمل الاسمية خلال 10 جمل من الأنماط المتتو عة كالآتي:

$$
\begin{aligned}
& \text { 1 ) أنا تاجر. } \\
& \text { 2) في القاعة مستمعون. } \\
& \text { 3) ورقتا هذا الدفتر ممزفتان. } \\
& \text { 4) هؤلاء الأطباء ماهرون. } \\
& \text { 5) (5ذا التاجر يده طويلة. } \\
& \text { 6) هذه المدينة شو ارعها و اسعة. } \\
& \text { 7) الكا الثواع دائما مزدحمة. } \\
& \text { 8) (7) مديرنا منتدد. } \\
& \text { 9) (أحمد ممرض من } \\
& \text { 10) (الدكاكين خالية }
\end{aligned}
$$

\begin{tabular}{|c|c|c|c|c|}
\hline النسبة المئوية & الترجمة غبر الصحيحة & النسبة المئوية & الترجمة الصحيحة & 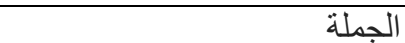 \\
\hline$\% 23$ & 17 & $\% 73$ & 58 & 1) أنا تاجر. \\
\hline$\% 75$ & 56 & $\% 25$ & 19 & 2) في القاعة مستمعون. \\
\hline$\% 39$ & 29 & $\% 61$ & 46 & 3) ورقتا هذا الدفتر مز قتان. \\
\hline$\% 31$ & 23 & $\% 69$ & 52 & 4) هؤلاء الأطباء ماهرون. \\
\hline$\% 20$ & 15 & $\% 80$ & 60 & 5) هذا التاجر يده طويلة. \\
\hline$\% 53$ & 40 & $\% 47$ & 35 & هذه المدينة شو ار عها و اسعة. \\
\hline
\end{tabular}

عند تصحيح ترجمة الطلبة للجمل الدذكورة أعلاها مستخرجا الترجمة الصحيحة وغير الصحيحة، قد تم حصول الجدول الآتي حسب الترجمة الصحيحة

و غير الصحيحة ونسبتهما المئوية. 


\begin{tabular}{|c|c|c|c|c|}
\hline$\% 19$ & 14 & $\% 81$ & 61 & 7) تلك الثو اع دائما مزدحمة. \\
\hline$\% 17$ & 13 & $\% 83$ & 62 & 8) مديرنا متشدد. \\
\hline$\% 29$ & 22 & $\% 71$ & 53 & 9) أحمد ممرض \\
\hline$\% 33$ & 25 & $\% 67$ & 50 & 10) الدكاكين خالية \\
\hline
\end{tabular}

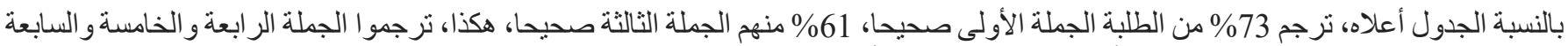

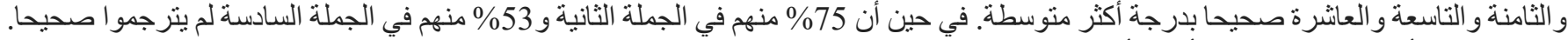
بناء على هذا، أن هذا البحث كثفت أنو اع أخطاء الترجمة عند ترجمة هذه الجمل العشر إلى اللغة التاملية كالتالي:

الإصر ار على ترجمة الكلمة العربية لكلمة واحدة بالتاملية، تقديم المذكر على المؤنث، الترجمة الحرفية، استخدام الألفاظ غير مناسبة للألفاظ العربية،

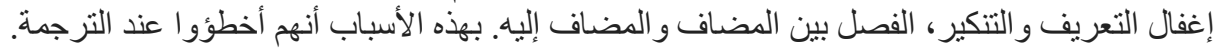

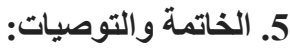

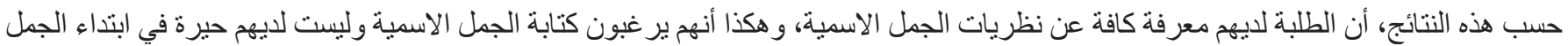

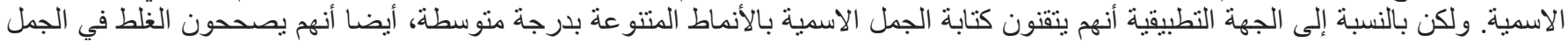

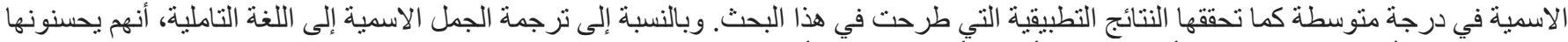
بدرجة متوسطة أيضا. بناء على هذا، أن هناك عدة أسباب أدت إليها، كما أشار ها الباحثون هشام ومناس و عليار (2017) كالآتي:

الاكتفاء بتعاليم المحاضر في قاعة المحاضرة دون البحث الموسع فيها. تحبر المعرفة الأساسية المتعلقة بفنون اللغة العربية مثل النحو و الصرف والإملاء. اقتصار رغبة الطلبة على كتابة الامتحان فقط دون التركيز على بناء الذات في تطوير اللغة العربية. عدم تعويد قر اءة الكتب و المجلات و الصحف العربية.

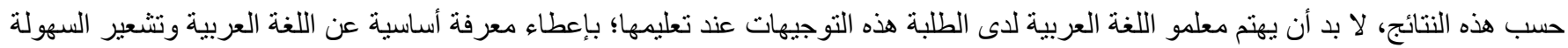

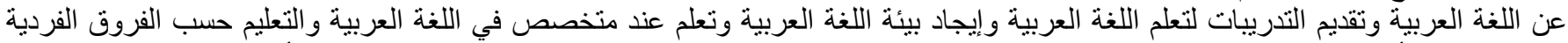

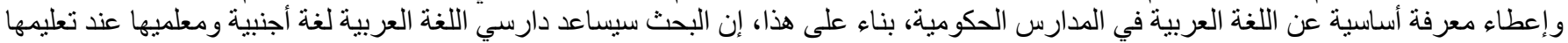

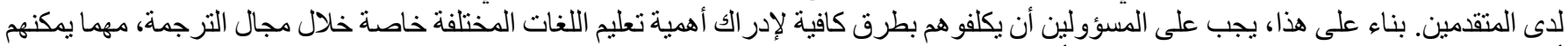

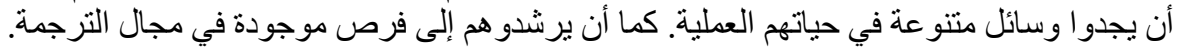

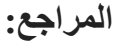

[1] [ ابن جني، أبو الفتح عثمان. (2006). الخصائص. تحقيق محمد على النجار، ط 1 1، بيروت: عالم الكتب.

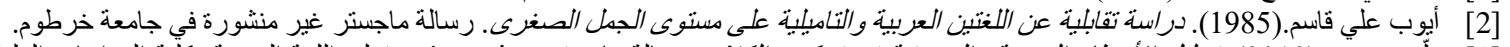

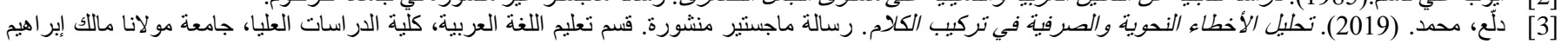

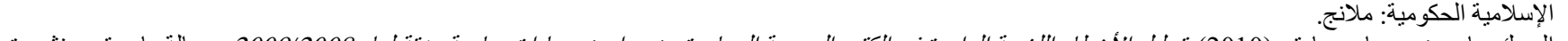
[4] الدويك، ياسمين مصباح صادقاق. (2010). تحليل الأخطاء اللغوية الواردة في الكتب الرسمية الصادرة من دواوين عمادات جامعة مؤنة لعام 2009/2008. رسالة ماجستير منشورة. جامعة مؤنة: عمادة الدراسات العليا.

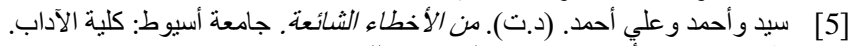

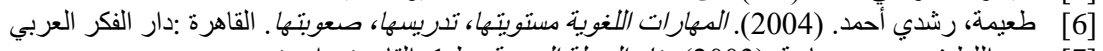

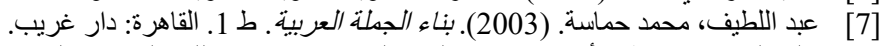

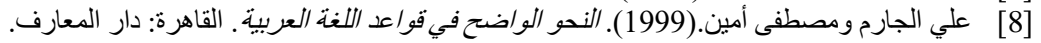

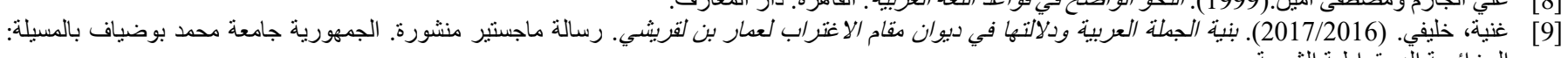
الجز ائرية الديمقر اطية الثعبية. [10] فتيحة، الصغير. (2015/2014). بناء الجملة العربية في ديوان نزار قباني أنعونجا ـ رسالة ماجستير منشورة. قسم اللغة و الأدب العربي، كلية الأدب و اللغات، جامعة العربي بن

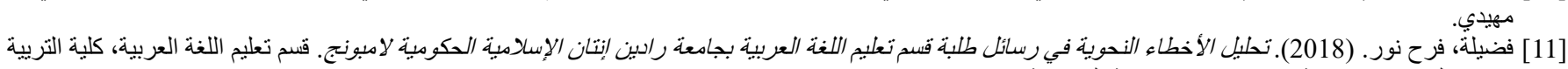
وإعداد المدرسين، جامعة رادين إنتان الإنسلامية الحكومية لامبونج.

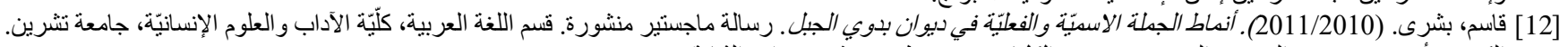

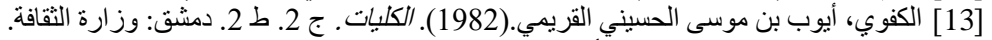
[14] لستاري، واندا. (2019). تحليل الأخطاء اللغوية في الإنشاء لطالاب الفصل الحادي عشر في قسم الدين بالددرسة العالية الحكومية. رسالة ماجنتير منشورة. قسم تعليم اللغة العربية،

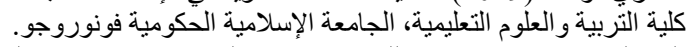

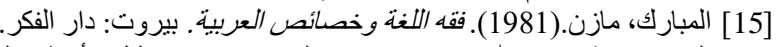
[16] النجران، عثمان عبدالله و جاسم، جاسم علي. (2013). تحليل الأخطاء الكتابية في بعض الظوانية الهر النحوية في كتابات الطلاب غير الناطقين بالعربية. الؤُتصر الدولي الثامن للغنة

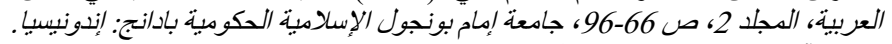
[17] هُشام وآخرون. (2017). استعمال الرو ابط في اللغة العربية لإى دارسيها كلغة ثانية على مستوى الجامعة نموذجا طلاب السنة الأولى من جامعة جنوب شرق سريلانكا.جامعة جنوب شرق سريلانكا: 